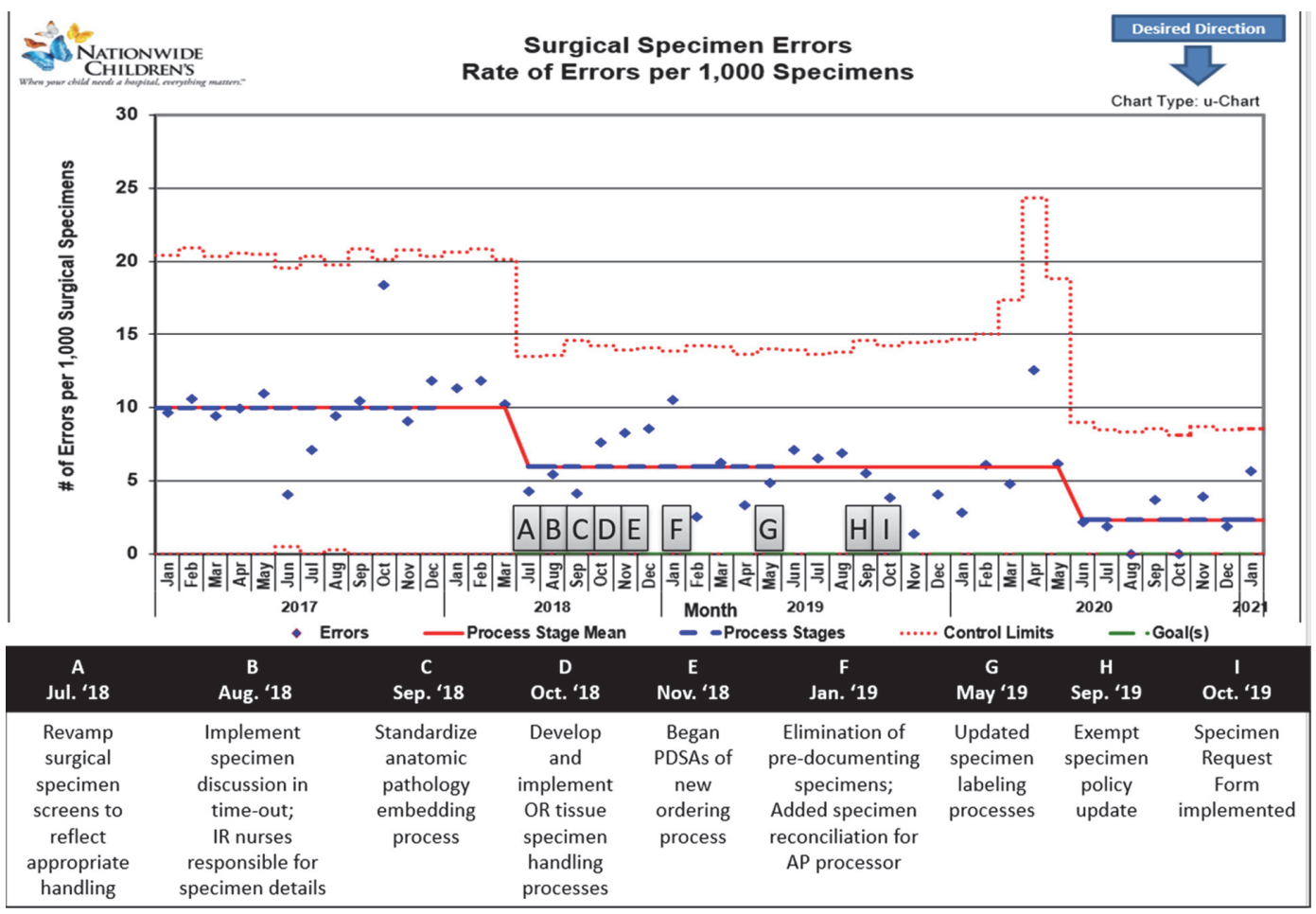

Abstract 15 Figure 3 The surgical specimen error outcome measure was tracked using a statistical process control U-Chart

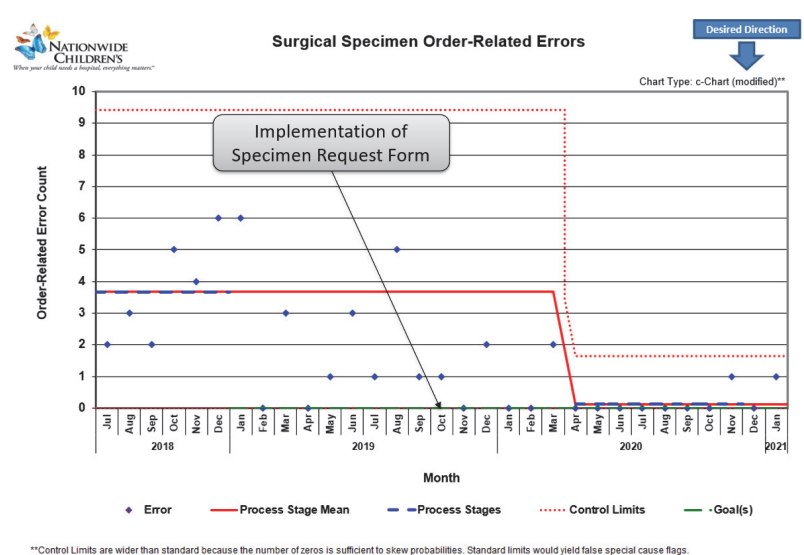

Abstract 15 Figure 4 This C Chart shows the count of order-related errors by month

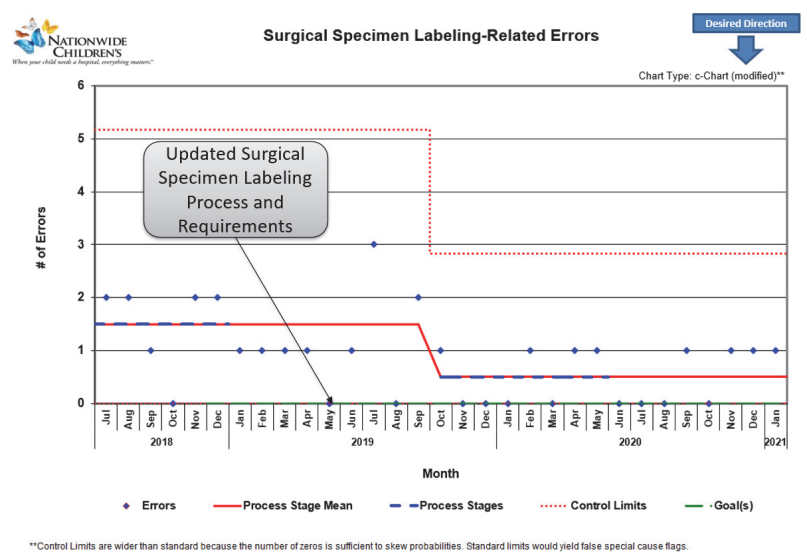

Abstract 15 Figure 5 This C-Chart shows the count of errors related to surgical specimen labeling each month and reconciliation processes, and optimized a method of communication between consultants, surgeons, operating room staff, and laboratory staff with the implementation of a Specimen Request Form within our electronic medical record system.

Results We identified 234 specimen errors in 33,962 surgical pathology specimens during the 46 months of data collection. After multiple PDSAs and implementation of interventions, the error rate decreased from 10 to 2.3 (figure 3 ) errors per 1,000 specimens $(p<.05)$. The mean rate of order errors (figure 4) decreased from 3.7 to 0.1 per month $(\mathrm{p}<.05)$, and the mean rate of labeling-related errors (figure 5) decreased from 1.5 to 0.5 per month $(\mathrm{p}<.05)$.

Conclusions A multidisciplinary project team - including representatives from nursing staff, surgical staff, radiology, medical consultation services, laboratory medicine, pathology, information technology, and QI services - implemented changes to specimen test requesting and intraoperative specimen handling with an associated reduction in errors to reduce potential patient harm involving surgical specimen errors. This presentation will demonstrate how applying these methods and interventions can be associated with a reduction in surgical specimen errors.

\section{VALUE IMPROVEMENT AT THE POINT OF CARE: AN INITIATIVE TO IMPROVE VALUE, FLOW, AND ACCESS}

${ }^{1}$ William Andrews, 'Poonam Gupta, ${ }^{2}$ lan McDonald, ${ }^{3}$ Salaheddin Arafa, ${ }^{4}$ Azhar Ali, ${ }^{4}$ Jeff Rakover. ${ }^{1}$ Hamad Medical Corporation Heart Hospital (HMC-HH) Quality Department; ${ }^{2}$ HMC-HH Department of Nursing; ${ }^{3}$ HMC-HH Department of Cardiology; ${ }^{4}$ Institute for Healthcare Improvement

\subsection{6/bmjoq-2021-IHI.16}

Background The value improvement (VI) approach was developed by the IHI and piloted at Raigmore Hospital, Scotland. 
It showed positive results by improving outcomes and reducing costs. Our team from a tertiary care hospital in Qatar used it to improve value in a clinically and geographically distinct context.

Objectives To evaluate the outcomes achieved from applying the VI methodology.

Methods The method is rooted in a framework that emphasizes standardization, continuous process improvement, and rightsizing capacity to demand. We collected and acted upon 3 types of indicators: performance, capacity (how resources are used), and financial measures. The main tools include a data box score (weekly updated table of data), a visual management board (showing run chart data, QI analyses, etc.), and weekly communication huddles to report progress and plan next steps.

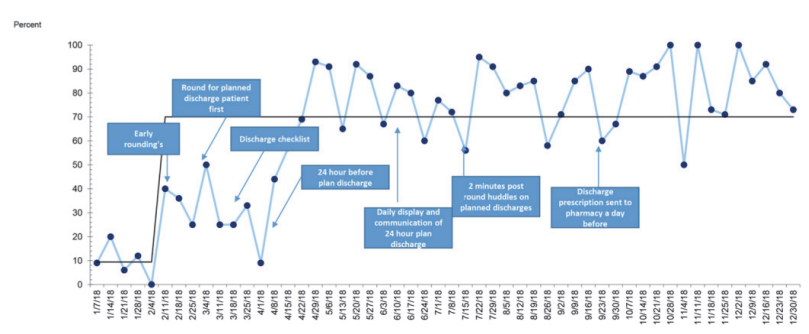

Abstract 16 Figure 1 Percentage of patients discharged before $1 \mathrm{pm}$

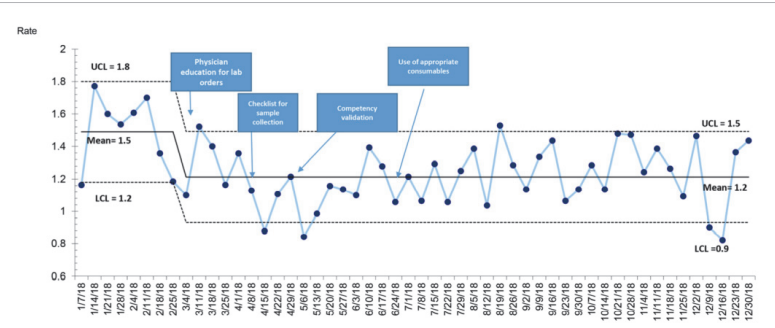

Abstract 16 Figure 2 Number of blood samples sent per patient per day

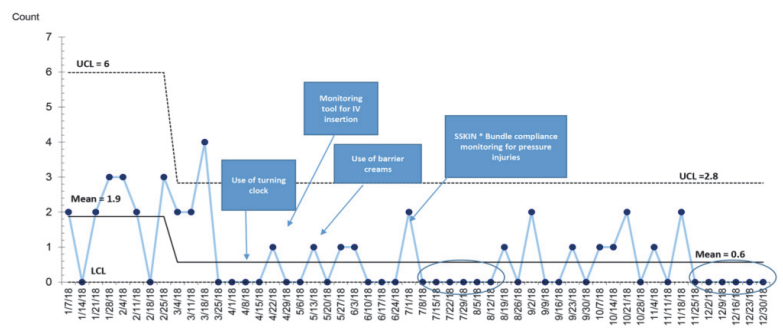

Abstract 16 Figure 3 Number of skin injuries, including pressure

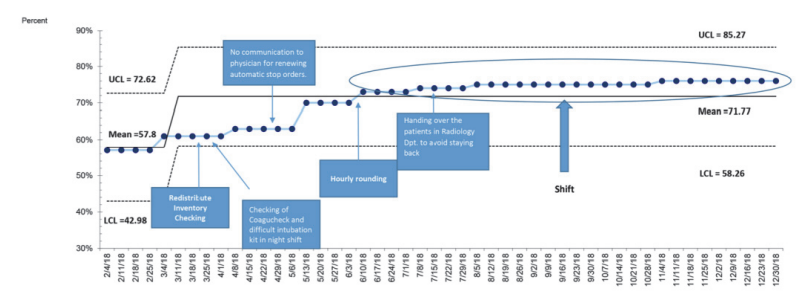

Abstract 16 Figure 4 Direct nursing care hours, morning shift

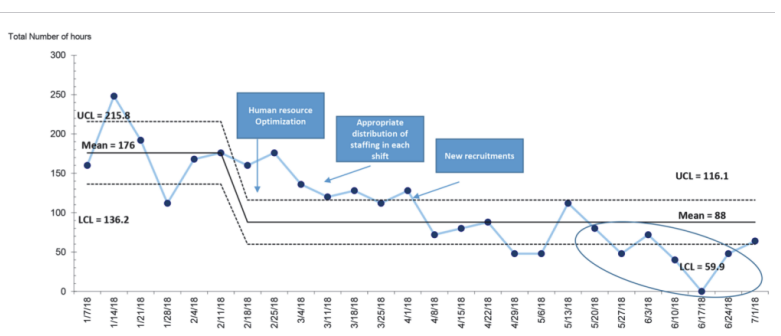

Abstract 16 Figure 5 RN overtime hours

Results Compared with a 4 - 8 week baseline data collection period, improvements included an increase in discharges before $13: 00$ by $61 \%$ (figure 1), reduction in blood samples per patient per day by $20 \%$ (figure 2), increase in nursing time spent in direct patient care by $18 \%$ (figure 4), reduction of skin injuries by $>50 \%$ (figure 3), completed VTE risk assessments increased 30\%, reduction of RN overtime hours by $50 \%$ (figure 5 ), lab sample rejection eliminated, and significant noise reduction in ICU. Currently more than fifty individual projects are completed or underway under the Value Improvement umbrella, the majority of which are showing improvements, often after only a few months.

Conclusions We found that the VI approach offered a systematic method for continuously improving the quality of care by focusing attention each week on safety, efficiency, and patient experience. The team improved numerous processes and outcomes resulting in a positive impact on patients and families and increased the engagement of staff in continuous improvement. In this way, we also improved our capacity to undertake and complete quality projects.

\section{IMPROVING EFFICIENCY IN SCHEDULING POST- DISCHARGE DERMATOLOGY FOLLOW-UP}

Maria Aleshin. Stanford Health Care

10.1136/bmjoq-2021-|HI.17

Background Timely post-discharge follow-up ensures safe inpatient/outpatient care transitions. Minimizing messaging to facilitate transitions is critical to staff well-being.

Objectives Improve the proportion of outpatient dermatology appointment scheduled within 2 weeks post-discharge to $>90 \%$; decrease staff messaging facilitating scheduling by $>25 \%$.

Methods Through process mapping dermatology care transitions (figure 1) we identified barriers to timely scheduling. We assessed impact of two interventions on post-discharge scheduling: video visits (VVs) initiated (March 2020) in response to COVID-19 and SmartPhrase intervention initiated (April 2021) in response to perceived high staff messaging rates (figure 2). We tracked time from discharge to scheduling of post-discharge appointment, percentage of patients scheduled $\leq 2$ weeks, and staff messaging from Jan 2019 through May 2021. Satisfaction was evaluated via stakeholder interviews.

Results Post-discharge VVs increased from 0\% to 64\% following adoption of telemedicine. The average number of days to schedule these improved from 31 days without VVs to 11 days with VVs; additional adoption of the SmartPhrase further reduced it to 6 days (figure 3). The percent of patients scheduled within 2 weeks improved from 79\% without VVs to $82 \%$ with VVs, and then to $90 \%$ with SmartPhrase adoption (figure 4). Compared to scheduling rates prior to 